PACS: 42.65.-k, 42.65.Pc, 42.70.Qs, 78.20.-e

\title{
All-optical signal processing in photonic structures with shifting bands
}

\author{
E.Ya. Glushko \\ V. Lashkaryov Institute of Semiconductor Physics, 45, prospect Nauky, 03028 Kyiv, Ukraine, \\ Kyiv Slavonic University, 9, Anry Barbusse str., 03150 Kyiv, Ukraine, \\ Phone: 52598 15, Fax: 52894 07, \\ E-mail: eugene.glushko@scientist.com
}

\begin{abstract}
We propose the principal scheme of all-optical adder based on the dependence of electromagnetic spectra in photonic bandgap materials containing optically nonlinear layers on the light signal intensity. The system consisting of periodical layered structure covered by optically nonlinear material is investigated and photonic structure behavior with changing intensity is calculated. Theoretical estimations of adder parameters are made for different nonlinear materials and frequencies of laser source.
\end{abstract}

Keywords: all-optical processing, nonlinear materials, photonic bandgap materials.

Manuscript received 30.06.04; accepted for publication 16.12.04.

\section{Introduction}

The awaited advantages of all-optical principles use in logical devices for optical computing, optical associative memories, and optical interconnections are connected with their higher operation frequencies in signal processing, small energy losses and practically unlimited possibilities to organize parallel operating the signals [1-5]. The all-optical ideology is used to mean the absence of electronic transforming signals at any stage of the process as well as spin or phonon mechanisms in signal processing. The reason of alloptical anticipated efficiency in obvious axiom that each signal transformation from one physical form into another decreases the common speed of signal passing through the device. By evaluations, the all-optical way in computing devices may have a great importance for the development of the next computer generation $[4,5]$.

We will consider all-optical signal processing based on the intensity depending photonic bandgap materials (PBG) containing optically nonlinear layers. Due to nonlinearity under illumination, both the bands position and local nonlinear modes position are shifted, which leads to a strong deviation in transmission and reflection of the light signal depending on its intensity. The all-optical adder task is transformation of a physical sequence of added signals to the logical sequence with corresponding shift of digital units. The idea of angular processing discussed in [6] for linear PBG materials is continued here as regard to nonlinear materials. It is important for our consideration that, when the intensity is changed, the nonlinearity causes the changes in the light signal angular distribution even at the steady input beam angles.

We will take into account also the fact that, in the logical adding process, there arise for some configurations of PBG system controlled by nonlinear insertions into the frequency-angular area sensitive to the signal transformation depending on its intensity. One more feature is that the adding process must be accompanied by necessary utilization of superfluous energy. For instance, let the physical sequence of two added signals has the form $(0,2,2,2, \ldots, 2)$ where numbers show the intensity magnitude. If the adder have transformed this sequence into the logical sequence $(1,1,1,1, \ldots, 0)$, then almost the half of the input energy have to be drawn aside. There exists also the problem of adder range precip by the rejected part of light beams. In this work, we discuss the way to solve the problem through the use of special precip appendix joining the each adder cell output and adsorbing the rejected energy.

The field structure for optically linear semiconductor, dielectric and mixed type 1D and 2D photonic crystals was investigated in previous works [6, 7]. It was shown that, for infrared optoelectronics of wavelengths in the interval $(1 \ldots 10) \mu \mathrm{m}$, the investigated structures GaAs/AlGaAs and GaAs/glass 1D layers had the characteristic frequency $\omega_{0}$ in the interval $(2 \ldots 20) \cdot 10^{14} \mathrm{~s}^{-1}$ and layer width ranging from 0.01 to 


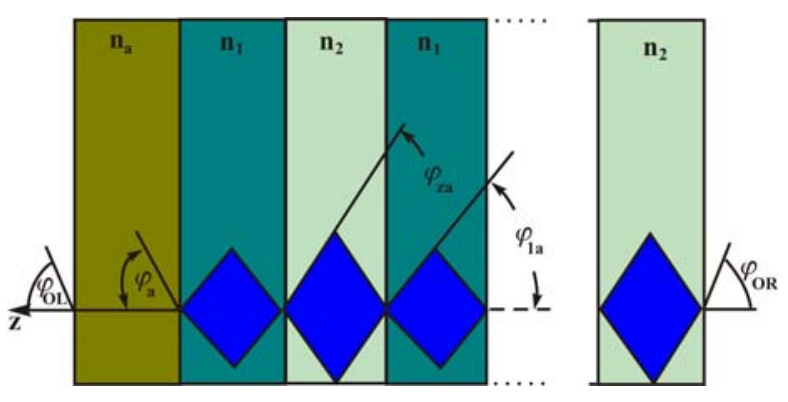

Fig. 1. Geometry of all-optical adder cell. The indexes $a$, 1, and 2 mark covering optically nonlinear layer and $\mathrm{PhCr}$ layers respectively, $n_{a}, n_{1}, n_{2}$ are refraction indexes, $\varphi_{a}, \varphi_{1 a}, \varphi_{2 a}$ are total intrinsic reflection angles, $\varphi_{0 \mathrm{~L}}, \varphi_{\mathrm{OR}}$ are left and right incidence angles, parallelogram areas show the corresponding total transmission cones.

$0.20 \mu \mathrm{m}$. In this case, there exists a possibility to operate by the mode population at a given frequency using special input fibres delivering external signals to each mode at separated angles $\varphi(i), i=1,2, \ldots, 16$. Calculated in [6] 32- and 16-periodic $\mathrm{GaAs}_{-} \mathrm{SiO}_{2}$ layered systems may serve as light reservoirs where two binary sequences experience the physical mixing. As to the signal adding, the problem was formulated there for a transformer having non-linear input-output characteristics of special view. Here, we will consider the all-optical device that transforms physically mixed sequence of signals into the logical sequence.

\section{Trapped modes inside the photonic crystal that contains nonlinear layers. The intrinsic problem.}

One-dimensional periodic layered structures exhibit bands and gaps in the spectrum of electromagnetic waves. Originally proposed by Yablonovitch [8] the PBG materials or the so-called photonic crystals $(\mathrm{PhCr})$ are attracting close attention due to a lot of possible applications. Layered hierarchical structures and the interferential nature of gap formation were investigated in [9] using the angular-frequency diagram method.

Regular well controlled photonic spectrum hierarchy produced by $\mathrm{PhCr}$ is the property perspective in optical computing, transforming and storing devices. Here, we will consider the physical principles and parameters of an optical chip operating due to the closed irradiation that is to say standing electromagnetic waves trapped inside the crystal by the total intrinsic reflection. In contrary to trapped irradiation, the transmitted one transfers the energy and momentum through the sample. Physically both types of waves may be differed in their properties, especially if one uses the analogy between quantum mechanical electronic states and the photonic ones [6]. Just kind of gaps corresponding to trapped waves has all grounds to be named as photonic gaps. In the case when the field source is located inside the $\mathrm{PhCr}$, the field spectrum for
1D layered structure is described by the system of interface boundary conditions [7,9] presented in Table, where $k_{s z}$ is the mode wavevector projection onto $z$ direction in $s$-material, $d_{s}$ is an appropriate layer width.

The 1D layered system under consideration (Fig. 1) consists of $N$ binary periods of alternating layers 1-2 and besides it includes one covering layer $a$ with the refraction index sensitive to the intensity of the incident light beam. The light transition through the system is determined by the Maxwell boundary conditions that form the system of $2 N+4$ equations. The matrix structure of the system is presented in Table where $L$ and $R$ columns mark the left and right media, the stroke means the returning wave running in opposite to $z$ direction, $I(\omega)$ is the light signal intensity. The intrinsic problem represents a situation when the source of the light is located inside the $\mathrm{PhCr}$, wave angles $\theta_{s}$ in each $s$-material belong to the total reflection range of the whole system: $\theta_{a}>\varphi_{a}$ and light energy don't leave the system. The electromagnetic field existing in the area are field eigenmodes satisfying the dispersion equation that describes the system determinant zeros. The generalized matrix-production form of the dispersion equation $[7,9]$ looks like:

$\left(Z_{l}-Y_{l}\right)\left(\begin{array}{l}\mu_{a}, v_{a} \\ \lambda_{a}, \mu_{a}\end{array}\right)\left(\left(\begin{array}{l}\mu_{1}, v_{1} \\ \lambda_{1}, \mu_{1}\end{array}\right)\left(\begin{array}{l}\mu_{2}, v_{2} \\ \lambda_{2}, \mu_{2}\end{array}\right)\right)^{N}\left(\begin{array}{l}Y_{r} \\ -Z_{r}\end{array}\right)=0$,

where each matrix represents a layer of the proper width $d_{s}$. Adding a new layer causes arising of its transfer matrix in the respective position. We will analyze one more geometry besides that shown in Fig. 1 with two ending nonlinear $a$-layers at both sides of the $\mathrm{PhCr}$. The production of binary structure layer matrixes under the power sign gives the transfer matrix of the $\mathrm{PhCr}$ period

$\left(\begin{array}{l}\mu_{1}, v_{1} \\ \lambda_{1}, \mu_{1}\end{array}\right)\left(\begin{array}{l}\mu_{2}, v_{2} \\ \lambda_{2}, \mu_{2}\end{array}\right)=\left(\begin{array}{l}\mu, v \\ \lambda, \bar{\mu}\end{array}\right)$,

$\mu_{s}=2 Y_{s} Z_{s} \cos \left(k_{s z} d_{s}\right)$,

$\lambda_{s}=2 i Z_{s}^{2} \cos \left(\theta_{s}\right) \sin \left(k_{s z} d_{s}\right), \quad s=a, 1,2$

$v_{s}=2 i Y_{s}^{2} \cos \left(\theta_{s}\right) \sin \left(k_{s z} d_{s}\right)$.

The nonlinear $a$-material should meet the demand of strong nonlinearity conjugated with small switching times in the picosecond area. As to the $\mathrm{PhCr}$ its nonlinearity must be insufficient and optical contrast between neighboring layers must be well expressed.

Represented in Fig. 2 is the diagram of nonlinear materials made in spirit of that published in [10]. The most attractive for optical signal processing area that unites the strong nonlinearity with small switching times lies in the left upper corner apart from the main sequence dots that occupy a direction from upper right corner to lower left one. We suppose that materials with lower refraction indexes are more preferred for the best exhibition of the nonlinear blooming properties. To demonstrate the general features of the effect, we will 


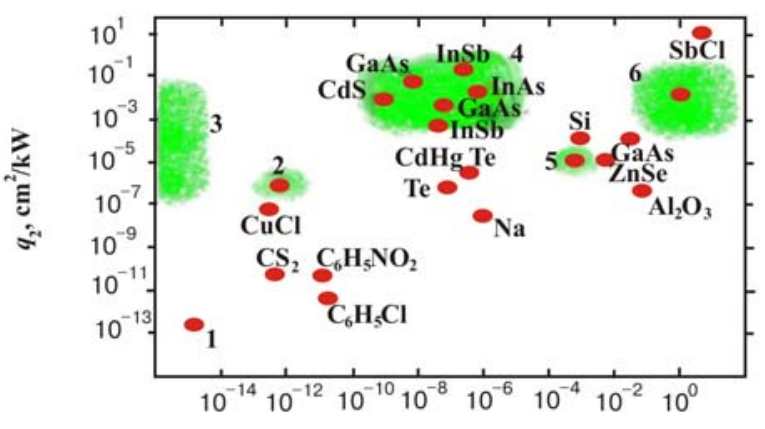

Fig. 2. The diagram of optically nonlinear materials. The $\chi^{(3)}-\tau$ diagram for nonlinear materials by [10]. 1 corresponds to quartz glass, 2 is doped glasses area, 3 shows area of super-nonlinear materials perspective for optical signal processing, 4 is area of electron nonlinearity in semiconductors, 5 shows dyes area, 6 marks liquid crystals area. Repeating materials are presented at the temperatures 10,77 , and $300 \mathrm{~K}$ from up to below (for instance, GaAS).

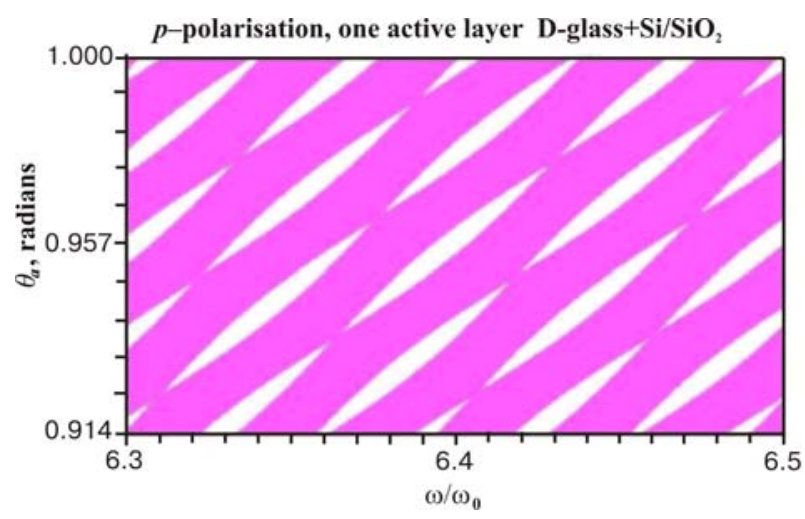

Fig. 3. Band angular-frequency diagram for glass-silicon/glass system. High-frequency range. Shaded areas are bands, $\omega_{0}=1.2415 \mathrm{eV}, d_{a}=10 \mu \mathrm{m}, d_{1}=30 \mu \mathrm{m}, d_{2}=20 \mu \mathrm{m}, I(\omega)=0$.

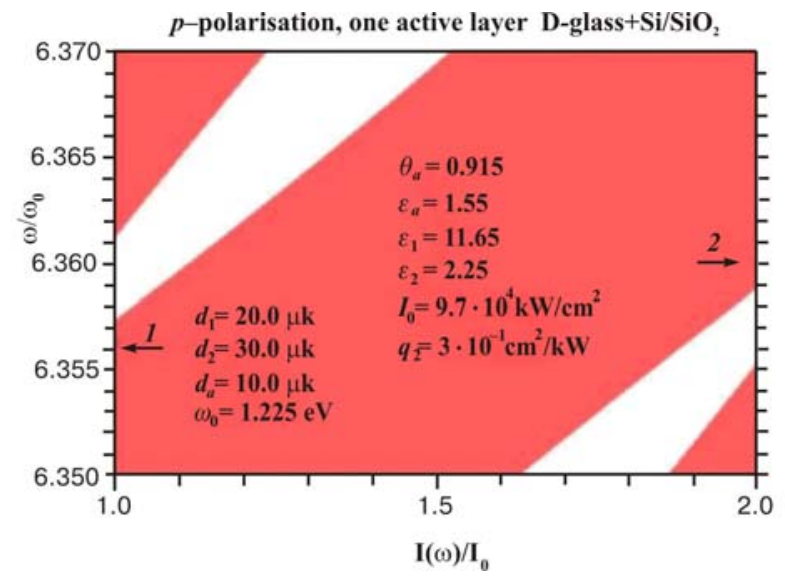

Fig. 4. Bandgap intensity dependence. Shaded areas are bands, $\omega_{0}=1.2415 \mathrm{eV} ; 1-\mathrm{R}$-scheme processing frequency $\omega_{\mathrm{a} 1}=6.356 ; 2-\mathrm{T}$-scheme processing frequency $\omega_{a 2}=6.360 \omega_{0}$, mode angle inside $a$-layer $\theta_{\mathrm{a}}=0.915$, beam "one" intensity $I_{0}=9.7 \cdot 10^{4} \mathrm{~kW} / \mathrm{cm}^{2}, q_{2}=3 \cdot 10^{-7} \mathrm{~cm}^{2} / \mathrm{kW}$. consider here the copper chlorine nonlinear layer controlling the optical properties of the structure. For cubic crystals, the approximate refractive index dependence vs external signal intensity looks like

$n_{a}(\omega)=n_{a 0}(\omega)+q_{2} I(\omega), \quad q_{2} \sim \chi^{(3)}(\omega)$.

Here, $n_{a 0}(\omega)$ is the refractive index without illumination, $I(\omega)$ is the intensity of light signal, $q_{2}$ is the nonlinearity coefficient taken from Fig. 2. The frequency dependence of refractive index was evaluated by the expression

$n_{a 0}(\omega)=n_{a 0}+n_{a 1}\left(\omega-\omega_{a 0}\right)$,

where $n_{a 0}=1.706, \quad n_{a 1}=0.1463, \omega_{a 0}=1.606 \mathrm{eV}$ for $\mathrm{CuCl}$ layer.

The diagram gives $q_{2}=10^{-7} \mathrm{~cm}^{2} / \mathrm{kW}$ that produces the needed $0.1 \ldots 1 \%$ modulation of the refraction index at beam intensities $I(\omega)=10^{5} \mathrm{~kW} / \mathrm{cm}^{2}$ having the order of the optical breakdown intensity.

More suitable materials based on silicon glasses doped by semiconductor impurities (Fig. 2, number 2) have more preferred nonlinearity coefficient $q_{2} \approx 10^{-}$ ${ }^{6} \mathrm{~cm}^{2} / \mathrm{kW}$, but corresponding switching times are worse. The requirements to materials forming the $\mathrm{PhCr}$ are described in the diagram by the area that lies lower than the main sequence diagonal or by the area characterized by large switching times. For optical signal processing purposes, it is convenient for the processes inside $\mathrm{PhCr}$ to be no more than $q_{2}<10^{-8} \mathrm{~cm}^{2} / \mathrm{kW}$ or if the switching time $\tau>10^{-6} \mathrm{~s}$. In both cases, the $\mathrm{PhCr}$ nonlinearity will be not essential in adopted area of signal intensities and signal processing frequencies.

We have calculated the eigenmodes of $\mathrm{TH}$ polarized plane waves trapped inside the $\mathrm{PhCr}$ by the total intrinsic reflection in the dependence on the incident angle, number of periods and layer widths. The activation of such states may be performed through the special input waveguides contacting with the nonlinear layer covering the $\mathrm{PhCr}$. The typical angular-frequency diagram calculated for a nonlinear material taken from the group 2 marked in Fig. 2 and silicon/glass layered structure as the $\mathrm{PhCr}$ is shown in Fig. 3. Shaded areas represent bands in the limit $N \rightarrow \infty$. The angular interval $0.914 \ldots 1.00 \mathrm{rad}$ corresponds to the total intrinsic range of this structure where the standing modes exist. Signal processing is performed at one of the angles from this interval and close to one of the band-gap-band zones. Presented figure gives a lot of possibilities to choose the operation points. Moreover, the undertaken calculations performed for different structures have shown a very precise scale effect of the band picture, especially in the lower part of the angular interval. With the accuracy better than $10^{-4}$, the band structure is not changed when transformations $\omega \rightarrow t \omega, d_{s} \rightarrow d_{s} / t$ are made where $t$ is constant and $s$ indicates materials. For the band structure shown in Fig. 3, the same will be for $\omega_{0}=0.12253 \mathrm{eV}$ and $d_{a}=100 \mu \mathrm{m}, d_{1}=300 \mu \mathrm{m}, d_{2}=200 \mu \mathrm{m}$. The reason is 
in transfer matrix (3) arguments of sine and cosine $k_{s} d_{s}$, where $k_{s}$ is proportional to $\omega$. The scaling property allows simply recalculate the photonic crystal parameters needed for the given laser frequency of the signals. For the laser signal wavelength $1.06 \mu \mathrm{m}$ that hits into Fig. 3 point $\omega_{l}=6.3 \omega_{0}$, the recalculation gives the nonlinear layer and silicon/glass $\mathrm{PhCr}$ parameters $d_{a}=1.51 \mu \mathrm{m}, d_{1}=3.02 \mu \mathrm{m}, d_{2}=4.53 \mu \mathrm{m}$. The order of bandwidths of assumed values is about $600 \mathrm{~cm}^{-1}$ and bandgaps $300 \mathrm{~cm}^{-1}$ (Fig. 3).

The intensity dependence of a selected spectral fragment calculated by (4) for the frequency interval $(6.35 \ldots 6.37) \omega_{0}$ is shown in Fig. 4. Here, we assign the light beam angle inside the $a$-layer $\theta_{a}=0.915$, the beam "one" intensity equals to $9.7 \cdot 10^{4} \mathrm{~kW} / \mathrm{cm}^{2}$, and nonlinearity coefficient $q_{2}=3 \cdot 10^{7} \mathrm{~cm}^{2} / \mathrm{kW}$. The left arrow indicates R-scheme processing at the convenient frequency $\omega_{a 1}=6.356 \omega_{0}$ corresponding to the chosen laser wavelength. The convenient $\mathrm{T}$-scheme processing frequency $\omega_{a 2}=6.360 \omega_{0}$. In both cases, the arrow position has to be chosen far enough from the band edge inside for the transferred signal and outside for the reflected one. The difference between $\mathrm{R}$ - and T-schemes is in the signal intensity "one" or "two" assigned for the reflected signal. The general demand for the number of $\mathrm{PhCr}$ periods $N>15$ is called to supply absolute reflection and transmission for chosen operation frequencies in the band edge vicinity. It is also of importance to consider the angular properties of the signal processing area. Taking into account the finite number of modes inside the band, the angle of processing $\theta_{a}$ should be corresponding to the possible maximal density of states.

Using (6), one can recalculate the system structure presented in Fig. 4 for energy unity $\omega_{0}=1.2253 \mathrm{eV}$ to the proportion that provides both processing schemes at the light signal wavelength $1.06 \mu \mathrm{m}$ that gives for R-scheme: $d_{a}=1.50 \mu \mathrm{m}, d_{1}=2.99 \mu \mathrm{m}, d_{2}=4.49 \mu \mathrm{m}$, gap $\Delta_{\mathrm{R}}=204.6 \mathrm{~cm}^{-1}$ and for T-scheme: $d_{a}=1.50 \mu \mathrm{m}$,

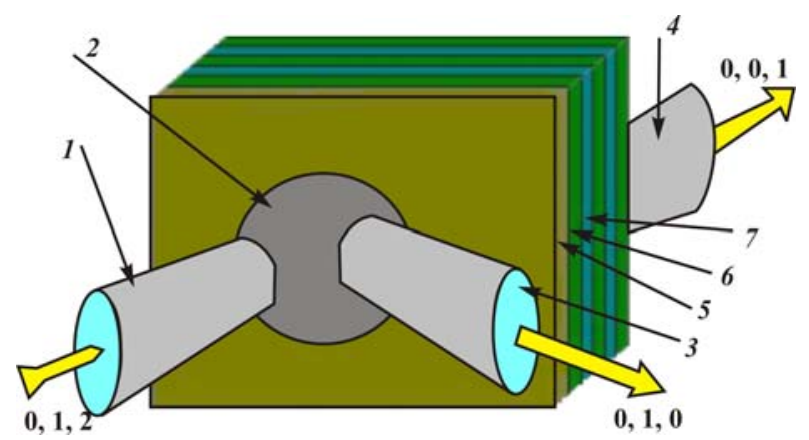

Fig. 5. Adder cell principal scheme. 1 - input waveguide; 2 input ledge; 3,4 - output waveguides (appendix lead isn't shown); 5 - nonlinear layer; 6,7 - two layers consisting the structure period. Yellow (light) arrows show light signals, digits show signal intensity for corresponding first, second or third position. $d_{1}=3.00 \mu \mathrm{m}, d_{2}=4.50 \mu \mathrm{m}$, gap $\Delta_{\mathrm{T}}=188.6 \mathrm{~cm}^{-1}$. The recalculation of Fig. 4 structure for $\mathrm{GaN}$ laser wavelength $0.36 \mu \mathrm{m}$ gives for R-scheme: $d_{a}=4.41 \mu \mathrm{m}$, $d_{1}=8.82 \mu \mathrm{m}, d_{2}=13.23 \mu \mathrm{m}$, gap $\Delta_{\mathrm{R}}=69.5 \mathrm{~cm}^{-1}$ and for $\quad$ T-scheme: $\quad d_{a}=4.41 \mu \mathrm{m}, \quad d_{1}=8.82 \mu \mathrm{m}$, $d_{2}=13.22 \mu \mathrm{m}$, gap $\Delta_{\mathrm{T}}=64.0 \mathrm{~cm}^{-1}$.

In fact, in our case the nonlinear influence of the active layer belongs to the kind of blooming effects. Though the covered active layer is thin as compared with the volume of $\mathrm{PhCr}$, the change of the signal intensity leads to the sufficient change in the incidence angle of the wave incident from the nonlinear layer onto the linear $\mathrm{PhCr}$. On the other hand, the kind of nonlinearity belongs to amplitude bistability effect.

\section{Adder cell schemes}

The system under consideration changes its reflectivity in dependence on signal intensity and may transmit bits to the neighbor major-order cell if the signal corresponds to " 2 ". We solve the task of an optical bit signal shift to the high-order digit position by using these two outputs for each input signal. One of the output signals intends for bit transport from junior position to major one if the physically added signal "2" arrives to the cell. Another one serves for both signals " 1 " and " 0 " pass through the cell without changing. There exist two ways to organize the shift of bit signal from low-order digit position to the high-order digit. In framework of R-scheme, the non-binary signal reflects from the cell. In the T-scheme, the signals of double intensity pass through the cell without changing. The cell structure for $\mathrm{T}$-scheme of signal processing is plotted in Fig. 5. The input signals " 0 ", "1" or " 2 " pass through the waveguide 1 directed relatively to the surface at the processing angle. The reflected signal arrives to output lead 3. The transferred signal passes through the output waveguide 4 . The input and output waveguides communicate with the cell by means of special ledges having the semi-spherical shape and situated on the outer sample surface. They aimed to supply the beam hitting inside the total reflection range.

In fact, the system considered above does not absorb the light beam energy. The only system action is to direct signals " 1 " and "2" through different outputs. The next problem is to reduce the intensity of the signal " 2 " passed through the cell to demanded "1". It can be achieved by the use of special beam splitters dividing doubled light signal into two equal parts. The splitter in the form of an appendix has to be joined to each adder cell output that corresponds to signal " 2 ". Depending on chosen processing scheme, it may be the reflection or transmission output. Another function of the beam appendix is in adsorbing of rejected energy. The maximal amount of the rejected energy during the adding process 
may reach a half of the input energy and the energy must be drawn aside.

In considered above cases described by Figs 1, 3-5 the system nonlinearity was presented in one outer layer. We have analyzed also the case with two nonlinear covering layers at both sides of $\mathrm{PhCr}$ when both output signal angles coincide. The calculated cell parameters were the same, if the number of $\mathrm{PhCr}$ periods remained big enough $(N \geq 20)$.

It is noteworthy that three-input adder cell design has the principal character. The design differs the adder cell from logical gates [12] where only two inputs are used. The adder third wave-guide input meant for the bit shifting into the side of the major digit. The analysis performed shows that transformation of the described adder cell into any of logical gates needs uniting the pair of inputs or blanking-off one of them.

\section{Signal processing inside the adder body}

Below we propose an essential development of all-optical adder presented in [6]. The perspective system for an adder cell includes one or several nonlinear layers covered the 1D photonic crystal. Due to sensitive areas in the system's angular-frequency diagram inside the whole reflection range the nonlinearity effect may lead to considerable change in light reflection and transmission. Both solitary local modes and band modes may be used. Due to the nonlinear shift depending on the input signal intensity 0,1 or 2 the cell proposed gives two output signals: in reflection 0,1 , and 0 respectively, and in transmission 0,0 , and 1 respectively. The combination of neighboring output signals allows performing the transformation of simple physical addition to logical one.

The binary summing scheme proposed before in [6] for two binary complicate optical signals having the structure of junior bits determined from characteristics of optical threshold amplifier. Demanded input-output characteristics of an amplifier that should transform a simple fractional optical signal into the simple integer signal of standard intensities include the existence of special falling areas. The presence of falling areas inside the input-output characteristics enabled to transform nonbinary signal (02) arising after the physical summation of two equal signals (01) and (01) into the binary one (10).

The modified approach is based on cell properties that allow differ signals " 1 " and " 2 ". There exist two close ways to organize binary adding of complicated signals built on the signal " 2 " reflection or transmission: R-scheme and T-scheme.

To realize the adder R-scheme let us situate the cells in columns and rows in such a way that unitary signals bringing the logical "one" let to move along rows without changing. Doubled signals due to the $a$-layer nonlinearity, chosen $\mathrm{PhCr}$ structure and frequency range are forced to reflect from the cell and redirect to the cell corresponding to the next digit and situated in the next column. The extra-intensity can be drawn aside by special appendix waveguides shown by red curls in Fig. 6.

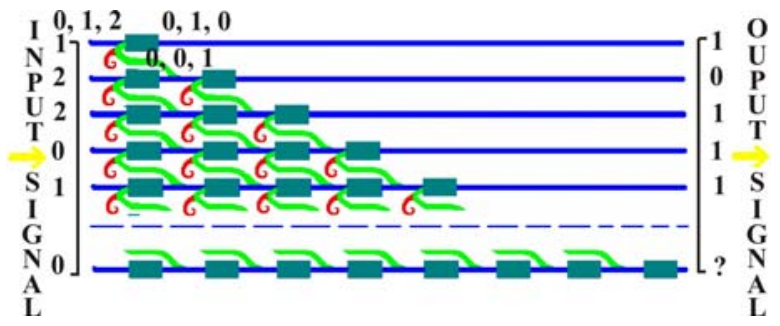

Fig. 6. R-scheme of physical signal transformation into logical sequence. The arrows show light signals, digits show signal intensity. $(0,1,2) \rightarrow(0,1,0)$ and $(001)$ in both outputs. The curl appendixes at reflection outputs reduce reflected 2 units twice.

Extended matrix of the boundary conditions system. Matrix elements: $Z_{s}=\cos \theta_{s} ; Y_{s}=-\varepsilon_{s} \sin \theta_{s} ; \varepsilon_{a}=\varepsilon_{a}(I(\omega))$;

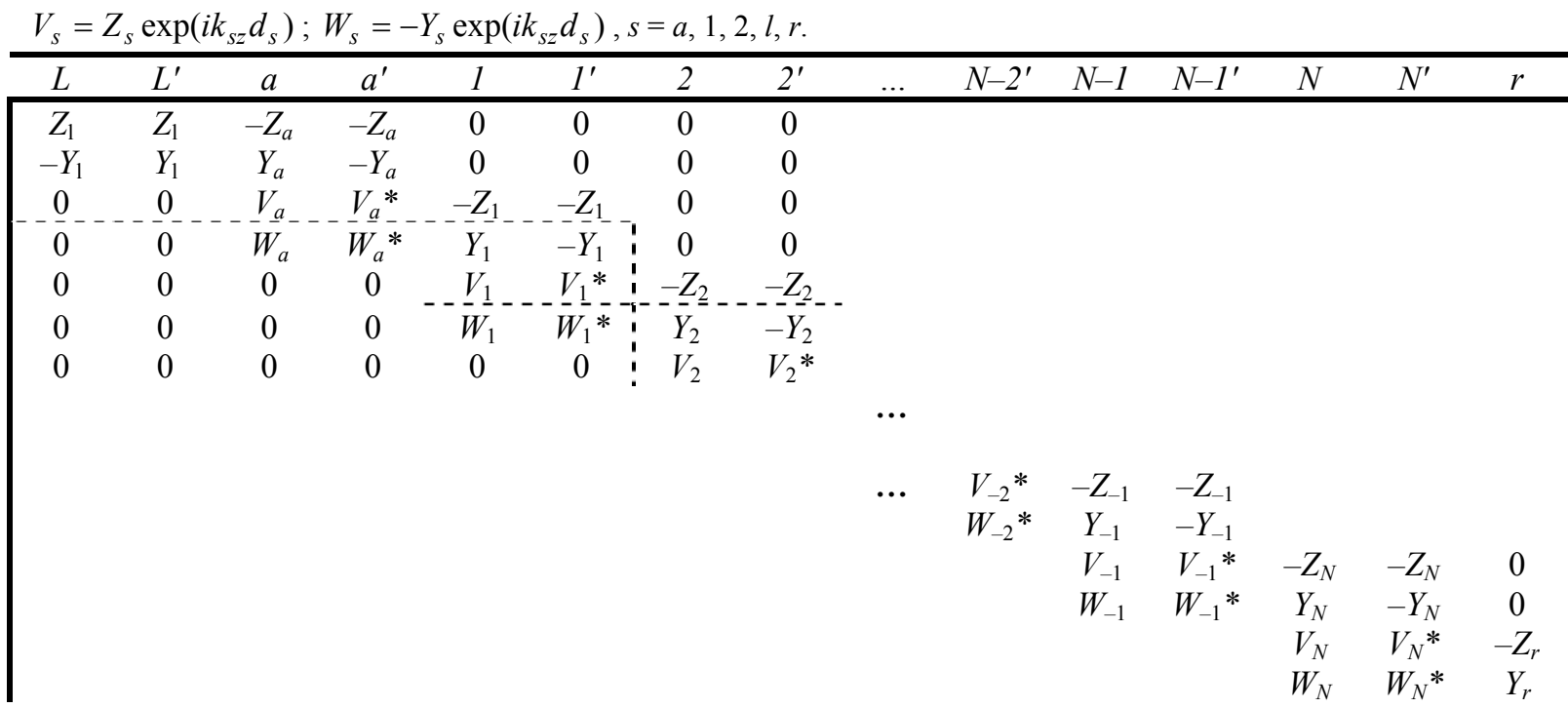


The adder T-scheme is characterized by another way of digit shifting. In $\mathrm{T}$-scheme, doubled signals pass through the cell without decreasing, whereas the physical "one" reflects from the cell body and is taken into account in this row digit. The transferred physical signal "two" must be detached into two signals "one" and one of them continues its way. In this case, the extra-intensity is also drawn aside by special appendix waveguides.

For both schemes, the number of cells in a column equals to the whole number of optical adder digit capacity. The digit hierarchy from junior to major is reflected in the adder in sequence directed from the top to down. Then the number of cells in a row depends on the row position in hierarchy. To process all three possible types of input physically added binary sequences containing " 0 ", " 1 " or " 2 " graded intensities the every next row should be one cell longer.

If $s$ is the adder's digit capacity, then for total cell number $S$ we have:

$S=\frac{(s+1) s}{2}$

For example, the 256-digit capacity adder should be consisted of 32896 cells connecting by optical channels in one of two possible schemes.

\section{Summary and discussion}

We have considered the features of all-optical signal processing based on mechanisms of nonlinear refraction index dependence on signal intensity. The details of adder cell design need more detail investigation of processes accompanied the signal transformation: heating, energy losses and energy redistribution between different channels. Important ones are geometry factors that determine the scale of system energy gaps from tens to thousands of inversed centimeters and mode energetic width. The width depends on both signal source and $\mathrm{PhCr}$ properties. The system imperfectness like deviating layer widths and refraction indexes ratio, interface boundaries roughness, light absorption and others cause nonzero eigenstate width. The mode width must be much less than the operation frequency distance from the nearest band edge. So, under similar conditions the more preferred are more thick layers ensured a higher accuracy in the widths ratio. A special contribution into the nonzero eigenstate width arises due to finite light beam section. This kind of state width restricts the cell miniaturization by the condition the beam section should be large in comparison with the light wavelength. The rough evaluation of a 256-digit device gives the order of $10 \mathrm{~cm}$ for its lateral sizes and $5 \mathrm{~cm}$ for the width if the operation wavelength is about a micrometer. The calculations performed here for materials taken from Fig. 2 give a lot of angular-frequency ranges suitable for $\mathrm{R}$ - or $\mathrm{T}$-signal processing schemes. The most effective area of parameters is the problem of further investigations. The suitable nonlinear materials for the controlling covering layer must be chosen in the left upper side of the diagram presented in Fig. 2. Other characteristics being equal to those of materials with smaller refraction indexes are more preferred.

The heat nonlinearity may be important in the acting adder due to the divergence of neighbouring material optical constants in the process of thermal expansion. To make the heating influence negligible, the difference between the thermal expansion coefficients of materials should be controlled. The dueto-heat relative divergence of refractive indexes for all material pairs have to be much less than that due to nonlinearity. In our case, it is easy to evaluate that the heating processes are weak for chosen materials. The heating problem could be solved by efficient organization of the adder topology when possible extraintensity may be leaded away from adder cells through the special appendix waveguides with following returning to the laser resonator for additional pumping.

\section{Acknowledgements}

The work was partially supported by the grant STCU2444.

\section{References}

1. M.F. Yanik, S. Fan, M. Sojiacic, and J.D. Jannopoulos, All-optical transistor action with bistable switching in a photonic crystal crosswaveguide geometry // Opt. Lett. 28, p. 2506-2508 (2003).

2. C. Coriasso, D. Campi, C. Caciatore, L. Faustiti, A. Stano, and C. Rigo, All-optical switching and pulse routing in a distributed feedback waveguide device // Ibid. 23, p. 183-186 (1998).

3. F.T.S. Yu and S. Jutamulia, Optical signal processing, computing, and neural networks, Wiley, New York (1998).

4. M.A. Karim, A.A.S. Awwal, Optical computing: an introduction, Wiley-IEEE Press (1992).

5. A.D. McAulay, Optical computer architectures: the application of optical concepts to next generation computers, Wiley-IEEE Press (1991).

6. S.L. Legusha and E.Ya. Glushko, Many-positional summator functioning on electromagnetic modes closed in a 1D photonic crystal // Proc. SPIE 5065, p. 47-53 (2002).

7. E.Ya. Glushko, S.L. Legusha, and A. Zakhidov, Electromagnetic quenching in 1D photonic crystals: closed modes and transferred irradiation // Ibid. p. 97-107 (2002). 
8. E. Yablonovitch, Inhibited spontaneous emission in solid state physics and electronics // Phys. Rev. Lett. 58, p. 2059-2063 (1987).

9. E.Ya. Glushko, Interference modes of an electromagnetic field in a layered structure // Optics and Spectroscopy 82, p. 304-308 (1997).

10. The tables of physical values, ed. by I.K. Kikoin, Moscow, Atomizdat, 1976 (in Russian).

11. H. Inoue, K. Tanaka, I. Tanahashi, T. Hattori, H. Nakatsuka, Ultrafast optical switching in a silver nanoparticle system // Jpn J. Appl. Phys. 39, p. 5132-5133 (2000).

12. M.F. Pereira Jr, M. Prado, and R. Sampaio, Many-body theory of all-optical quantum well logic gates // Semiconductor Physics, Quantum Electronics \& Optoelectronics 5 (3), p. 312-315 (2002). 